\title{
Recurrence-based analysis of barrier breakup in the standard nontwist map
}

Moises S. Santos, Michele Mugnaine, José D. Szezech, Antonio M. Batista, Iberê L. Caldas, Murilo S. Baptista, and Ricardo L. Viana

Citation: Chaos 28, 085717 (2018); doi: 10.1063/1.5021544

View online: https://doi.org/10.1063/1.5021544

View Table of Contents: http://aip.scitation.org/toc/cha/28/8

Published by the American Institute of Physics

Chaos

An Interdisciplinary Journal of Nonlinear Science

Fast Track Your Research. Submit Today! 


\title{
Recurrence-based analysis of barrier breakup in the standard nontwist map
}

\author{
Moises S. Santos, ${ }^{1}$ Michele Mugnaine, ${ }^{1}$ José D. Szezech, Jr., ${ }^{2}$ Antonio M. Batista, ${ }^{2, a)}$ \\ Iberê L. Caldas, ${ }^{3}$ Murilo S. Baptista, ${ }^{4}$ and Ricardo L. Viana ${ }^{5}$ \\ ${ }^{1}$ Pós-Graduação em Ciências, Universidade Estadual de Ponta Grossa, 84030-900 Ponta Grossa, Paraná, Brazil \\ ${ }^{2}$ Departamento de Matemática e Estatística, Universidade Estadual de Ponta Grossa, 84030-900 Ponta Grossa, \\ Paraná, Brazil \\ ${ }^{3}$ Instituto de Física, Universidade de São Paulo, 05508-900 São Paulo, São Paulo, Brazil \\ ${ }^{4}$ Institute for Complex Systems and Mathematical Biology, University of Aberdeen, SUPA, Aberdeen AB24 3UE, \\ United Kingdom \\ ${ }^{5}$ Departamento de Física, Universidade Federal do Paraná, 80060-000, Curitiba, Paraná, Brazil
}

(Received 5 January 2018; accepted 22 March 2018; published online 28 August 2018)

\begin{abstract}
We study the standard nontwist map that describes the dynamic behaviour of magnetic field lines near a local minimum or maximum of frequency. The standard nontwist map has a shearless invariant curve that acts like a barrier in phase space. Critical parameters for the breakup of the shearless curve have been determined by procedures based on the indicator points and bifurcations of periodical orbits, a methodology that demands high computational cost. To determine the breakup critical parameters, we propose a new simpler and general procedure based on the determinism analysis performed on the recurrence plot of orbits near the critical transition. We also show that the coexistence of islands and chaotic sea in phase space can be analysed by using the recurrence plot. In particular, the measurement of determinism from the recurrence plot provides us with a simple procedure to distinguish periodic from chaotic structures in the parameter space. We identify an invariant shearless breakup scenario, and we also show that recurrence plots are useful tools to determine the presence of periodic orbit collisions and bifurcation curves. Published by AIP Publishing. https://doi.org/10. $1063 / 1.5021544$
\end{abstract}

Many relevant problems in fluids and plasmas can be described, in the language of Hamiltonian dynamical systems, by the use of nontwist maps. The common feature of these systems is the presence of shearless invariant curves, which affect the transport properties in phase space. Actually, the shearless curve behaves like a barrier separating trajectories in the phase space. However, even after breakdown of the shearless curves, the rate of chaotic transport depends on the system parameters. We analyse the barrier breakup in the standard nontwist map by means of the determinism, a quantification from recurrence plots. In this work, we also show that determinism can be used to identify the island chain collisions and separatrix reconnection.

\section{INTRODUCTION}

The standard nontwist map (SNM) was introduced by Del-Castillo-Negrete and Morrison; ${ }^{1}$ it violates the twist condition along the shearless curve. ${ }^{2}$ The twist condition asserts the nondegenerancy of the frequencies. This map presents interesting phenomena such as island chain collisions and separatrix reconnection.

Magnetic field lines in toroidal plasma devices can be described by area preserving nontwist maps. ${ }^{3}$ Martins and collaborators ${ }^{4}$ used the SNM to study transport barriers in plasmas confined in tokamaks. Studies concerning internal transport barriers are important to improve plasma

a)Electronic mail: antoniomarcosbatista@gmail.com confinement and stability properties. The SNM also appears in fluids, as in the description of transport in quasigeostrophic flows. ${ }^{1}$ Transport properties in nontwist maps were investigated by Szezech and collaborators. ${ }^{5}$ They showed that the barrier escape time and barrier transmissivity have sensitive dependence on the parameters. The identification of barriers has been done by means of the finite-time Lyapunov exponents or the finite-time rotation number. ${ }^{6}$ One of the pioneer methods to detect breakup barriers was based on residue criteria and applied in the standard map ${ }^{7}$ and nontwist map. ${ }^{2}$

We investigate the possibility of using the recurrence quantification analysis (RQA) technique to characterise the SNM dynamics, in particular, for studying barrier breakup. RQA was proposed by Zbilut and Webber, ${ }^{8,9}$ and it has been widely utilised for the investigation of complex systems. ${ }^{10,11}$ RQA was considered in studies about the complexity of dynamics in discharged magnetised plasma. ${ }^{12}$ The determinism is a measure calculated from the RQA. It is the percentage of recurrence points which form diagonal lines in the recurrence plot. Determinism analysis has been applied to different science fields. ${ }^{12}$

In Hamiltonian systems, the use of recurrence time is a useful tool to distinguish chaotic from quasi-periodic orbits. Slater's theorem states that if there is a quasi-periodic orbit, at most three different recurrence times are expected; otherwise, the orbit is chaotic. ${ }^{13}$ In the literature, this theorem was applied successfully to detect the presence of barriers in Hamiltonian systems, for instance, the twist map and the non-twist map. ${ }^{14}$ The use of the recurrence analysis was also done to determine the breakup of shearless torus in 
non-Hamiltonian systems. ${ }^{15}$ Moreover, a common behaviour of these Hamiltonian systems is the presence of stickiness effect. The existence of stickiness causes long chaotic trajectories around the neighbourhood of some islands. Zou and collaborators ${ }^{16}$ showed how RQA provides a useful way to detect the dynamical transitions from chaotic to sticky regime.

Our main result is to show that determinism analysis, i.e., the quantification of determinism provided by the RQA approach, can be used as a diagnostic of barrier breakup in the SNM as well as the island chain collisions and separatrix reconnection. The study concerning the breakup of shearless invariant tori is used to describe transport barriers in magnetic field lines in toroidal plasma devices. ${ }^{17}$ In this work, we identify in the parameter space by means of determinism analysis not only the barrier breakup but also the island chain collisions and separatrix reconnection.

This paper is organised as follows: Sec. II introduces the SNM and the transport barrier. In Sec. III, the determinism analysis is proposed for the identification of relevant phenomena such as the barrier breakup. Section IV exhibits collision scenarios. In Sec. V, we draw our conclusions.

\section{STANDARD NONTWIST MAP}

Nontwist maps are area-preserving maps which violate the twist condition along an invariant curve, known as shearless curve, in phase space. We consider the SNM proposed and described in Ref. 18 as

$$
\begin{aligned}
& x_{n+1}=x_{n}+a\left(1-y_{n+1}^{2}\right), \\
& y_{n+1}=y_{n}-b \sin \left(2 \pi x_{n}\right),
\end{aligned}
$$

where $a$ and $b \in \mathbb{R}$ are parameters, $x \in[-1 / 2,+1 / 2)$ and $y \in \mathbb{R}$ are phase space coordinates. The variable $n=$ $0,1,2, \ldots, N$ denotes the discrete time. Figure 1 shows the phase space for some values of the SNM control parameters. In Figs. 1(a)-1(c), we observe a separation of the phase space into two regions, one below and the other above. A shearless curve (blue line) is responsible for diking the transport (a)

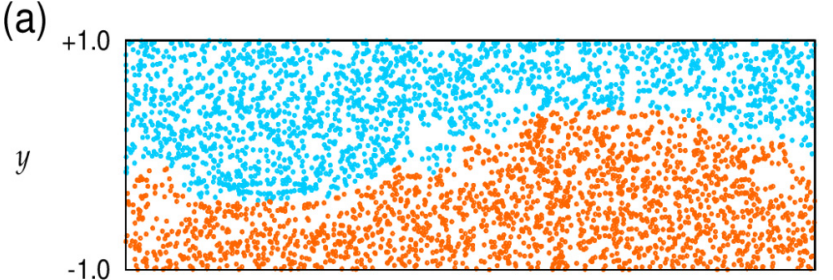

(b)

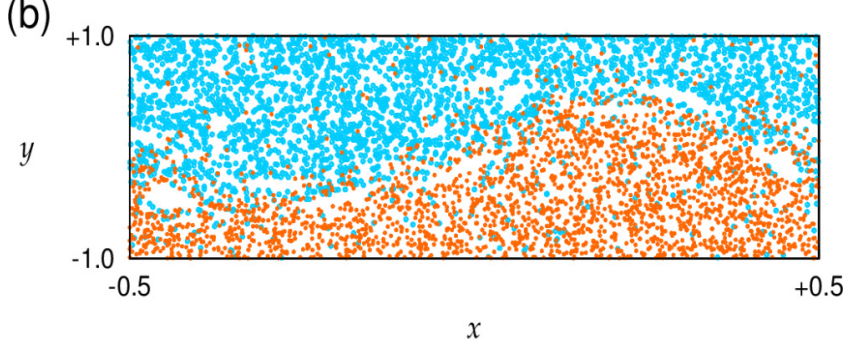

FIG. 2. Transport barriers of the SNM for (a) $a=0.455$ and $b=0.8$, (b) $a=0.455$ and $b=0.847$. The region in blue (orange) colour represents $10^{3}$ initial conditions on the line $y=1(y=-1)$ iterated by $N=2 \times 10^{4}$.

of field lines between the regions. There are chains of islands separated by nontwist invariant tori. In Fig. 1(d) there is no more shearless barrier curve, and as a consequence the two regions merge in the phase space.

Figure 2 shows the phase space of orbits in different colours, starting from several initial conditions. In our simulations, we consider $N=2 \times 10^{4}$ iterations and $10^{3}$ initial conditions on the line $y=1(y=-1)$ to obtain the blue (orange) orbits. In Fig. 2(a), we consider the same parameters as in Fig. 1(c), the shearless curve can be estimated to be located at the boundaries between the two different coloured regions. In Fig. 2(b), generated with the same parameters as in Fig. 1(d), the shearless curve does not exist and the transport of trajectories throughout the phase state is now possible.

Nontwist maps have been considered to describe magnetic field lines in toroidal plasma in stellarators and tokamaks. ${ }^{19}$ With regard to tokamaks, the appearance of internal transport barrier in experiments can be analysed by means of nontwist maps. ${ }^{20}$ They have also used in studies (a)

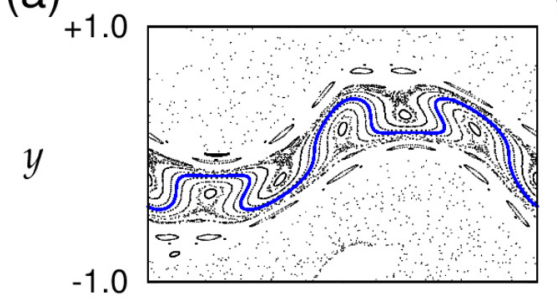

(c)

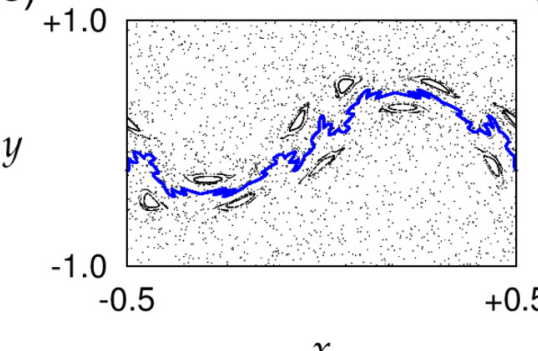

$x$ (b)

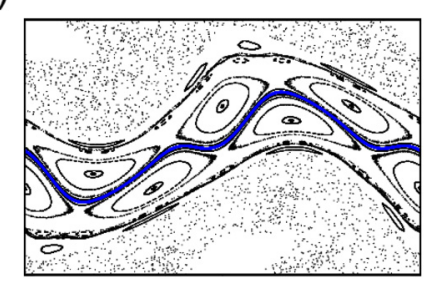

(d)

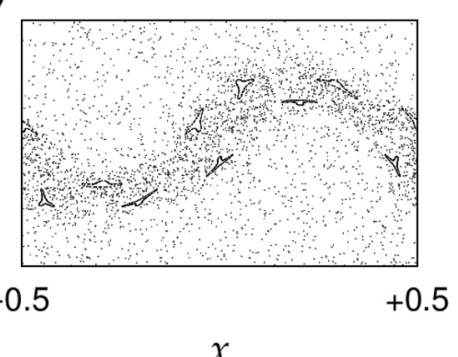

FIG. 1. Phase space of the SNM for (a) $a=0.354$ and $b=0.6$, (b) $a=0.364$ and $b=0.6$, (c) $a=0.455$ and $b=0.8$, and (d) $a=0.455$ and $b=0.847$. The blue line represents the shearless curve obtained by the evolution of the IP $(1 / 4, b / 2)$ as the initial condition in Eq. (1). 
about flows in fluid dynamics. ${ }^{21}$ Moreover, in mathematics, their importance is due to the fact that around the nontwist region they do not behave according to the KAM and Poincare-Birkhoff theorems. ${ }^{22}$

\section{RECURRENCE ANALYSIS}

The recurrence plot (RP) is a visualisation of a square matrix where a dot is placed at $(i, j)$ whenever $\vec{x}_{i}$ is nearby to $\vec{x}_{j} .{ }^{23-25}$ The RP can be mathematically expressed as

$$
R P_{i, j}=\Theta\left(\varepsilon-\left\|\vec{x}_{i}-\vec{x}_{j}\right\|\right),
$$

where $\vec{x}_{i} \in \mathbb{R}^{m}(i, j=1 \ldots k), k$ is the number of possible states $\vec{x}_{i}$ in $m$-dimensional space, $\varepsilon$ is the return radius $(\varepsilon=0.05),\|\cdot\|$ indicates the Euclidean norm, and $\Theta(\cdot)$ is the Heaviside function. The interesting patterns observed in RPs led the authors in Ref. 8 to develop recurrence quantification analysis (RQA) to quantify the structures present in the RPs. Thus, several diagnostics ${ }^{26,27}$ can be obtained from Eq. (2), for example, recurrence rate, laminarity, determinism, etc. Following Ref. 27, determinism is quantified by

$$
\mathrm{DET}=\frac{\sum_{l=l_{\min }}^{k} l P(l)}{\sum_{l=1}^{k} l P(l)},
$$

where $P(l)$ represents the probability distribution of diagonal lines of length $l\left(l_{\min }=2\right)$ present in the recurrence plot. A diagonal line of length $l$ indicates whether there are two timely separated pieces of the time-series that remain $\varepsilon$-close by a time of $l$. The more deterministic a system is, the longer the diagonal lines will be. Stochastic systems have very short or no diagonal lines at all (i.e., no two adjacent points can be seen in the diagonal). Therefore, the determinism measure is the rate between diagonal lines with length larger than $l_{\min }$ and all the points with no adjacent neighbour $(l=1)$. The more (less) predictable a system is, the closer to 1 (zero) will be the measure DET.

Figure 3 exhibits the average value of the determinism in the colour bar [(DET)] for $N=10^{4}$ times. We split $N$ in intervals (windows) to compute the determinism. Each window has 400 points $(k=400)$ of the trajectory and there are
300 points overlapping between two consecutive windows. Thus, each window provides a DET value and the mean determinism is calculated at the end of time. In Figs. 3(a)-3(c) it is possible to identify the transport barrier in the shearless region that are characterised by high values of the determinism measure DET. Comparing the results of Fig. 3 with Fig. 1, we verify that the regions of Fig. 3 marked by red and yellow correspond to the regions quasi-periodicity (islands) and the blue corresponds to the regions chaoticity. In Fig. 3(d), we see the mixing of chaotic orbits due to the breakup of the transport barrier. Comparing Fig. 3(c) with Fig. 1(c), we verify that the determinism allows us to identify those barriers in the phase space by noticing that the barriers have a distinct dissimilar colour representation appearing in a level of colour far from both red and dark blue extremes.

\section{COLLISION SCENARIOS}

In bidimensional maps, such as the SNM, the invariant curves represent absolute barriers in the phase space. ${ }^{14,17}$ Pioneering methods to identify the barrier break can be found in Refs. 7 and 28. Greene ${ }^{7}$ explored the relation between KAM surfaces and periodic orbits through the residue. The Greene's residue criterion can be used to determine the existence or not of invariant tori. Those methods need a long time iteration $\left(N \approx 10^{6}\right)$ to obtain the shearless curve by using one of the following indicator points (IPs):

$$
\mathbf{z}_{\mathbf{0}}^{( \pm)}=( \pm 1 / 4, \pm b / 2), \quad \mathbf{z}_{\mathbf{1}}^{( \pm)}=(a / 2 \pm 1 / 4,0),
$$

as initial condition for Eq. (1). The indicator points are fixed points of some of the symmetries of the SNM that are on the shearless curve whenever it is not broken. ${ }^{29}$

Figures 4(a) and 4(b) exhibit at the boundary between the two colours the parameters for the breakup of the last shearless curve in the parameter space $a \times b$. The colour bar identifies barrier break by means of the method shown in Ref. 17. We iterate the indicator points (IPs) for a long time $\left(N=10^{6}\right)$ and a given pair of values of $a$ and $b$, then if these trajectories scape of a given interval $(|y|>20)$, the barrier is broken (yellow region), otherwise, the barrier still exists (blue region). (a)

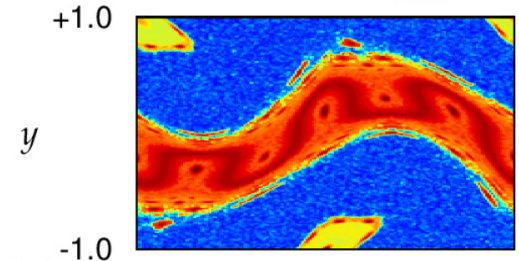

(c)

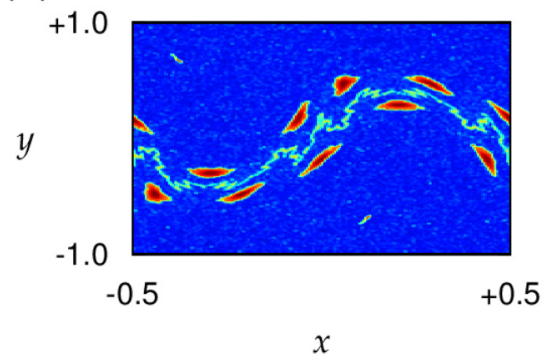

(b)

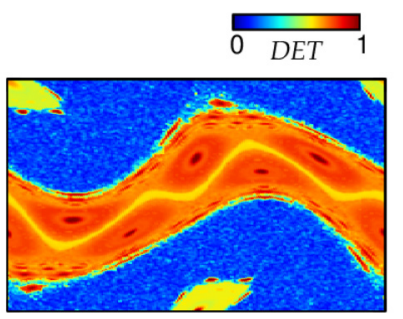

(d)

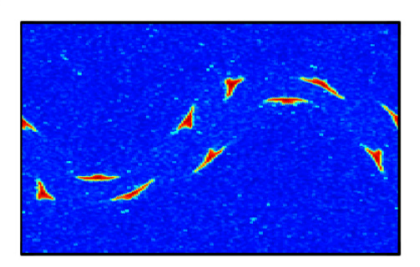

$-0.5$
FIG. 3. Determinism phase space of the SNM for (a) $a=0.354$ and $b=0.6$, (b) $a=0.364$ and $b=0.6$, (c) $a=0.455$ and $b=0.8$, and (d) $a=0.455$ and $b=0.847$. 
(a)

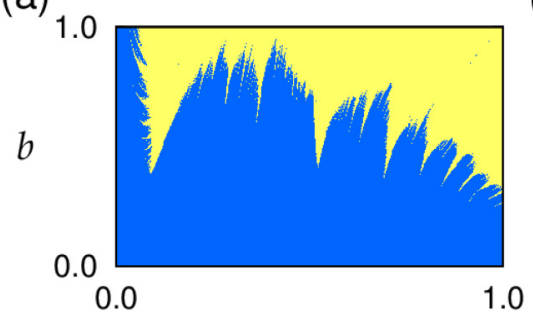

(c)

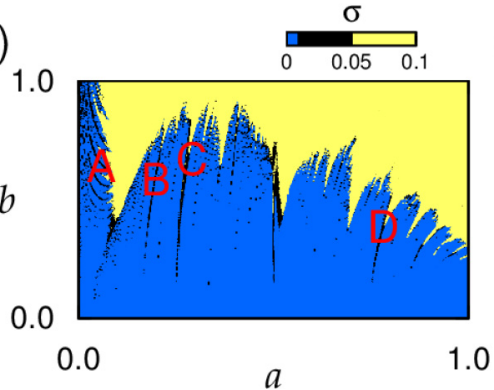

(b)

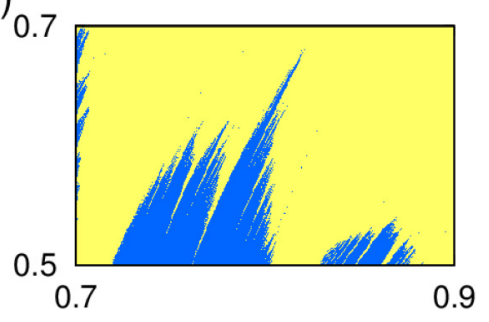

(d)

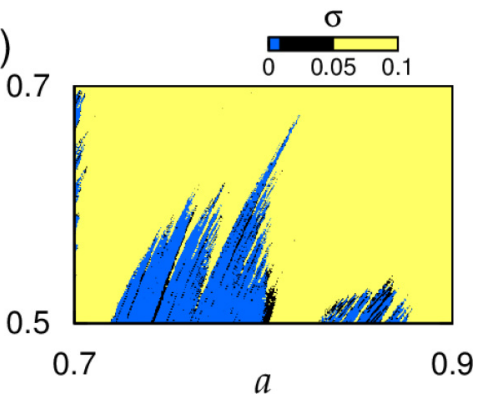

FIG. 4. Barrier breakup of the SNM using two methods. Figures (a) and (b) are calculated through the IP according to Ref. 17, where the blue (yellow) region represents the existence (breakup) of the shearless curves. Figures (c) and (d) are obtained using standard deviation of the determinism, where the black region corresponds to the bifurcation curves.
In this article, we introduce another procedure to determine the shearless curve breakup. This procedure is based on the recurrence plot analysis and is simpler to be applied than the previous procedures. Due to the fact that the determinism values change on the shearless curve, as shown in Fig. 3, we calculate the standard deviation of the determinism to identify the existence of barriers. The standard deviation of the determinism is given by

$$
\sigma=\sqrt{\left\langle(\mathrm{DET})^{2}\right\rangle-\langle\mathrm{DET}\rangle^{2}} .
$$

In the parameter spaces of Figs. 4(c) and 4(d), we show by colour coding the value of $\sigma$ computed using IP as initial condition. The presence of the barrier (or not) is obtained through the analysis of the RQA measure (DET). The colour bar represents the standard deviation of the determinism for short time $\left(N=10^{4}\right)$ of the IP. We utilise moving windows of length $k=400$, where 300 points overlapped between two consecutive windows. Each window provides a DET value and the standard deviation is calculated at the end of time. The blue and yellow regions not only are similar to regions found in Figs. 4(a) and 4(b) (showing the value of DET in colour code) but are also obtained with less iterations, as compared to if we had reproduced these figures considering the values obtained only for the determinism measure DET. The novelty is the black region that corresponds to parameters representing when the system bifurcates. The blue and yellow regions represent parameters for which the shearless curves exist or do not, respectively. The bifurcation parameter curve lying within the black region appears for parameters that cause a collision between up and down periodic orbits on a symmetry line when the SNM parameters are varied. ${ }^{2}$ The points A, B, $\mathrm{C}$, and D in Fig. 4(c) indicate some values of $a$ and $b$ where the bifurcation curves occur (black region) in the parameter space.

We plot in Fig. 5 the phase space for the values of $a$ and $b$ according to points A [Fig. 5(a)], B [Fig. 5(b)], C [Fig. 5(c)], and D [Fig. 5(d)], as shown in Fig. 4(c). The phase spaces display the dipole formation after the hyperbolic collision. ${ }^{2}$ In Fig. 5(a), it is possible to observe the collision of central islands, and similar behaviour occurs in Figs. 5(b)-5(d), but for orbits of different periods. (a)

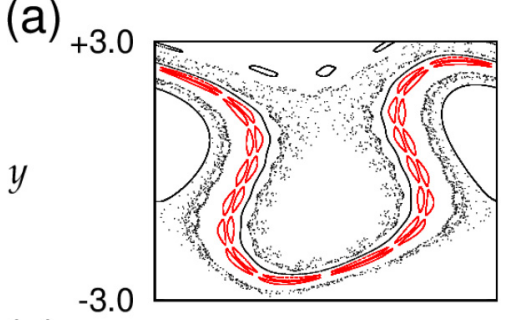

(c)

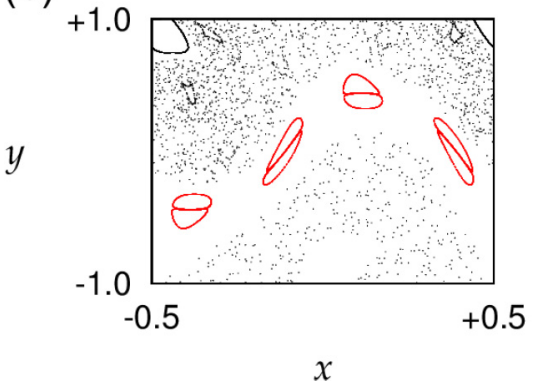

(b)

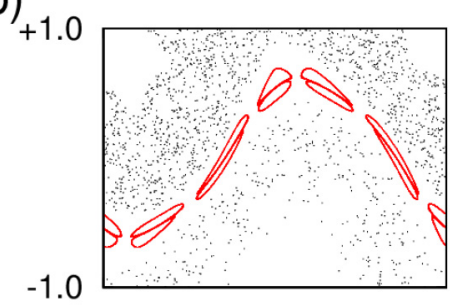

(d)

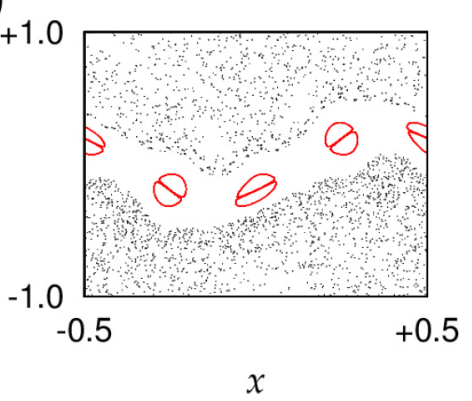

FIG. 5. Periodic orbit collision in SNM for (a) $a=0.0413$ and $b=0.71$, (b) $a=0.21$ and $b=0.69$, (c) $a=0.28$ and $b=0.69$, and (d) $a=0.782$ and $b=0.39$. These parameters values correspond to the points $\mathrm{A}, \mathrm{B}, \mathrm{C}$, and D, respectively, showed in Fig. 4(c). 
(a)

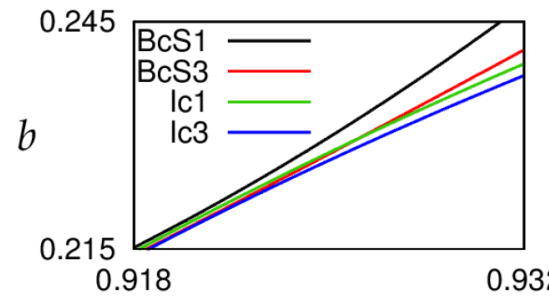

(c)

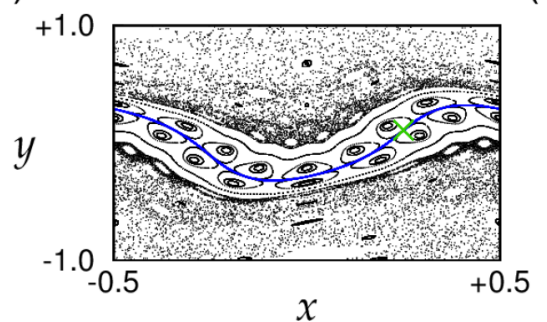

(b)

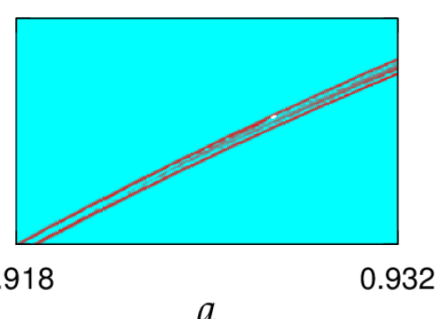

(d)

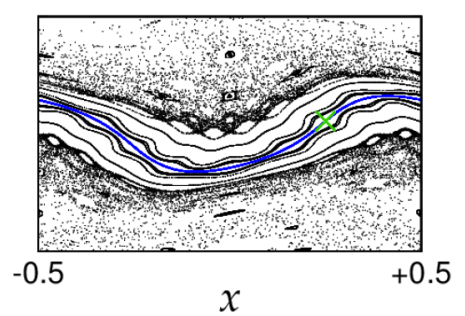

FIG. 6. (a) Indicator and bifurcation curves, and (b) standard deviation of the determinism. Phase space for (c) $a=$ 0.91856 and $b=0.21504$, and (d) $a=0.91856$ and $b=$ 0.21640 showing collision and annihilation, respectively, of the periodic orbits $(q=7$ and $p=8)$, where the green point represents the indicator and the blue line denotes the shearless curve.
An orbit with period $p$ is an orbit $M^{p}\left(x_{i}, y_{i}\right)=\left(x_{i}+q, y_{i}\right)$, where $q$ is an integer number, consequently, the winding number $\omega=q / p$ is rational. For orbits with the same stability type for both the up and down orbits, namely, orbits with even period, the symmetry lines $S_{1}=\{(x, y) \mid x=0\}$ and $S_{3}=\left\{(x, y) \mid x=a\left(1-y^{2}\right)\right\}$ can be used to obtain the numerical bifurcation threshold. ${ }^{30}$ For even-period orbits, the bifurcation values for the parameters $a$ and $b$ (indicator curve Ic), it can be obtained from the solution of $M^{p} \mathbf{z}_{\mathbf{j}}{ }^{( \pm)}=\mathbf{z}_{\mathbf{j}}{ }^{( \pm)}$and $M^{p / 2} \mathbf{z}_{\mathbf{j}}^{( \pm)}=\mathbf{z}_{\mathbf{j}}^{(\mp)}$, where $\mathbf{z}_{\mathbf{j}}{ }^{ \pm}$are the indicator points defined in Eq. (4). ${ }^{30}$

Figure 6(a) exhibits the indicator curves Ic1 for $S_{1}$ and Ic3 for $S_{3}$, and it also shows the curves $\mathrm{BcS} 1$ and $\mathrm{BcS} 3$ that are numerically calculated by means of the winding number, for a small region of the parameter space. The winding number is given by $\omega \equiv \lim _{i \rightarrow \infty}\left(x_{i} / i\right)$, where an $x$-coordinate is "lifted" from $\mathbb{T}$ to $\mathbb{R}$. We consider $i=t_{r}+t_{s}$, where the variable $t_{r}=3 \times 10^{6}$ is the transient time and $t_{s}=2 \times 10^{5}$ is the time required for the limit to exist. $\omega$ is calculated on the symmetry lines $S_{1}=\{(x, y) \mid x=0\}$ and $S_{3}=\left\{(x, y) \mid x=a\left(1-y^{2}\right)\right\} .^{30}$

We obtain the Ic1 and Ic3 curves by means of the standard deviation of the determinism, as shown in Fig. 6(b) (red lines). For a fixed $a$ value and two distinct $b$ values, the SNM presents collision [Fig. 6(c)] and annihilation [Fig. 6(d)] of the periodic orbits for the red bottom and top lines, respectively. Therefore, through the determinism measure we can estimate the location of the breakup barrier in phase space as well as the bifurcation curves (collision of elliptic orbits and collision of nonsymmetric hyperbolic orbits) in the parameter space for orbits with even period found in the SNM. Then, determinism analysis can be used as a tool to identify the bifurcation threshold in the SNM with period-even orbits.

\section{CONCLUSIONS}

We consider the SNM that is an area-preserving map and violates the twist condition. This map is used in studies concerning barriers in plasma and fluids. The SNM shows the breakup of invariant tori and separatrix reconnection.

In this work, we propose the use of determinism analysis to study the breakup of shearless invariant curve and bifurcation threshold for orbits in the SNM. We show that the determinism allows us to identify barriers in the phase space. By means of the standard deviation of the determinism we find the barrier breakup region in the parameter space. Moreover, the determinism analysis, when compared with other methods, needs less time iteration to compute the indicator points that belong to the shearless curve.

Through the standard deviation of the determinism, we identify in the parameter space not only the barrier breakup region but also the region where the bifurcation curves occur. The bifurcations are due to the collision of elliptic orbits and collision of nonsymmetric hyperbolic orbits. In addition, we performed our analysis with DET, but we also computed and verified that similar results can be achieved based on other RQA measures, such as recurrence rate and laminarity.

In future works, we plan to analyse how geometric characteristics of recurrence network analysis ${ }^{31}$ for nontwist systems could detect the breakup of shearless invariant curve.

\section{ACKNOWLEDGMENTS}

We wish to acknowledge the support from CNPq, CAPES, and FAPESP.

${ }^{1}$ D. Del-Castillo-Negrete and P. J. Morrison, Phys. Fluids A 5, 948 (1993). ${ }^{2}$ D. Del-Castillo-Negrete, J. M. Greene, and P. J. Morrison, Physica D 91, 1 (1996).

${ }^{3}$ W. Horton, H. B. Park, J. M. Kwon, D. Strozzi, P. J. Morrison, and D. I. Choi, Phys. Plasmas 5, 3910 (1998).

${ }^{4}$ C. G. L. Martins, R. E. de Carvalho, I. L. Caldas, and M. Roberto, J. Phys. A 43, 175501 (2010).

${ }^{5}$ J. D. Szezech, Jr., I. L. Caldas, S. R. Lopes, R. L. Viana, and P. J. Morrison, Chaos 19, 043108 (2009).

${ }^{6}$ J. D. Szezech, Jr., I. L. Caldas, S. R. Lopes, P. J. Morrison, and R. L. Viana, Phys. Rev. E 86, 036206 (2012).

${ }^{7}$ J. M. Greene, J. Math. Phys. 20, 1183 (1979).

${ }^{8}$ J. P. Zbilut and C. L. Webber, Jr., Phys. Lett. A 171, 199 (1992).

${ }^{9}$ C. L. Webber, Jr. and J. P. Zbilut, J. Appl. Physiol. 76, 965 (1994). 
${ }^{10}$ M. S. Santos, J. D. Szezech, Jr., A. M. Batista, I. L. Caldas, R. L. Viana, and S. R. Lopes, Phys. Lett. A 379, 2188 (2015).

${ }^{11}$ M. S. Santos, J. D. Szezech, F. S. Borges, K. C. Iarosz, I. L Caldas, A. M. Batista, R. L. Viana, and J. Kurths, Chaos Solitons Fractals 101, 86 (2017)

${ }^{12}$ V. Mitra, B. Sarma, A. Sarma, M. S. Janaki, A. N. S. Iyengar, N. Marwan, and J. Kurths, Phys. Plasmas 23, 062312 (2016).

${ }^{13}$ N. Slater, Proc. Cambridge Philos. Soc. 63, 1115 (1967).

${ }^{14}$ C. V. Abud and I. L. Caldas, Physica D 308, 34 (2015).

${ }^{15}$ E. G. Altmann, G. Cristadoro, and D. Pazó, Phys. Rev. E 73, 056201 (2006).

${ }^{16}$ Y. Zou, M. Thiel, M. C. Romano, and J. Kurths, Chaos 17, 043101 (2007).

${ }^{17}$ A. Wurm, A. Apte, and P. J. Morrison, Braz. J. Phys. 34, 1700 (2004).

${ }^{18}$ K. Fuchss, A. Wurm, A. Apte, and P. J. Morrison, Chaos 16, 033120 (2006).

${ }^{19}$ A. Apte, A. Wurn, and P. J. Morrison, Chaos 13, 421 (2003).

${ }^{20}$ R. Balescu, Phys. Rev. E 58, 3781 (1998).
${ }^{21}$ D. Del-Castillo-Negrete and M.-C. Firpo, Chaos 12, 496 (2002).

${ }^{22}$ J. D. Meiss, Rev. Mod. Phys. 64, 795 (1992).

${ }^{23}$ J. P. Eckmann, S. O. Kamphorst, and D. Ruelle, Europhys. Lett. 4, 973 (1987).

${ }^{24}$ N. Marwan, Eur. Phys. J. Spec. Top. 164, 3 (2008).

${ }^{25}$ M. C. Romano, M. Thiel, J. Kurths, and C. Grebogi, Phys. Rev. E 76, 036211 (2007).

${ }^{26}$ W. S. Chen, Physica A 390(7), 1332 (2011).

${ }^{27}$ N. Marwan, M. C. Romano, M. Thiel, and J. Kurths, Phys. Rep. 438, 237 (2007).

${ }^{28}$ J. Laskar, C. Froeschlé, and A. Celleti, Physica D 56, 253 (1992).

${ }^{29}$ S. Shinohara and Y. Aizawa, Prog. Theor. Phys. 100, 219 (1998).

${ }^{30}$ A. Wurm, A. Apte, K. Fuchss, and P. J. Morrison, Chaos 15, 023108 (2005).

${ }^{31}$ Y. Zou, R. V. Donner, M. Thiel, and J. Kurths, Chaos 26, 023120 (2016). 